record for most tests administered per shift increased from 55 for one lane to 112 for two lanes (figure 1).

Conclusions Lessons learned here about heat safety, pathogen safety, and hot zone training can be applied to all drive thru testing sites. With this ongoing pandemic, it is wise to look for ways to improve test sites as well as potential vaccination sites.

\section{RHEUM SERVICE: IMPROVING VIRTUAL CARE DURING COVID-19}

Joseph Carson, Stephanie Gottheil. London Rheumatology; Western University, Canada

\subsection{6/bmjoq-2020-IHI.9}

Background During COVID-19, rheumatology outpatients need timely access to care while social distancing. Video consults have potential to improve virtual assessments, however, some patients and providers are apprehensive about using this technology.

Objectives Provide delightful and effective video consults for $90 \%$ of new patients by July 1 .

Methods We redesigned video appointments to create a seamless virtual experience. PDSA Series 1 identified improvement opportunities with a process map, fishbone, and driver diagram. PDSA Series 2 tested and implemented change ideas: digital appointment confirmations, reminders, and forms; video consults with limited pre-call testing; digital reports, requisitions, and messaging. PDSA Series 3 refined changes by decreasing reminders, increasing pre-call tests, and adding backup video platforms. Outcome measures were: 1)\% consults by video, 2)\% requesting more video appointments. Process measures were: 1) pre-call tests completed, 2) technical difficulties. Our balance measure was $\%$ virtual diagnoses modified after in-person visits. We collected data over ten weeks and emailed anonymized patient surveys one week after video consults. We analyzed data with run charts and descriptive statistics.

Results We scheduled 135 new consults: 120 (89\%) video, 14 (10\%) phone, and 1 (1\%) office. Twenty-one patients (16\%) did not own a video-enabled device. Pre-visit, 12 patients $(10 \%)$ participated in pre-call testing. Video consults were initiated for $97 \%$ of scheduled patients; of these, $6 \%$ suffered technical difficulties, requiring a switch to phone or another video platform. Surveys were completed after 40\% (48/120) of video visits: $68 \%$ of patients wanted another video appointment; 28\% were 'not sure'; 4\% declined. Virtual diagnoses stayed the same for $84 \%(32 / 38)$ of patients with follow-up in-person assessments.

Conclusions While video consults proved effective for most patients, sociodemographic and technological barriers prevented others from participating. Next steps include improving access to video-enabled devices and providing more pre-visit training to reduce these barriers.

\section{USE OF MANAGERIAL EPIDEMIOLOGY BY HEALTHCARE} LEADERS IN AMBULATORY SETTINGS

Chantelle Schenning. Walden University, United States

10.1136/bmjoq-2020-IHI.10
Description Despite many health reforms, the United States continues to struggle with limited healthcare access, exponential healthcare costs, and poor quality of care. Overcoming these challenges requires healthcare leaders' effective navigation of industry transformation towards population health and a shift in patient volume to ambulatory healthcare settings. Research has demonstrated that the use of managerial epidemiology, an application of epidemiology tools and principles to management decision-making within healthcare organizations, can better serve the health of the population and could improve the triple aim of inadequate access, high costs, and poor quality. However, the adoption of this practice is weak and its utilization by current healthcare leaders has not yet been studied. Diffusion of innovation theory framed this qualitative study to understand the perspectives of ambulatory healthcare leaders on using managerial epidemiology within their leadership approach as well as understand the spread of this practice and associated barriers. Twelve healthcare leaders participated in semistructured interviews. Findings from openaxial coding of the interview data indicated managerial epidemiology is critical and validated the importance of managerial epidemiology for impacting the triple aim, population health, and overall system performance. Additionally, this study provided steps to accelerate the adoption and highlighted the use of managerial epidemiology during a pandemic, which has worldwide implications for improving health and performance of healthcare globally therefore promoting social change.

\section{FRAMING EQUITY, DIVERSITY \& INCLUSION EFFORTS ACROSS THE CONTINUUM OF MEDICAL EDUCATION USING IHI MODEL FOR IMPROVEMENT}

Deborah Simpson. Advocate Aurora Health, USA

\subsection{6/bmjoq-2020-|HI.11}

Background Medical education (ME) must create equitable, diverse, and inclusive (EDI) training environments for our learners, faculty, and staff and equitable care for the patients they serve. As ME leaders spanning students to CME and libraries, we are accountable for addressing structural 'isms' in all forms (eg, race, gender-identity, religion).

Objectives Our purpose is to be intentional and public in our actions to address EDI across the continuum of medical education using the IHI Model for continuous improvement. We have 3 specific objectives, one each at the micro, meso, and macro levels.

Methods Each education department leader provided their current EDI activities and focal areas for future work along with proposed measures. These interventions were then reviewed to identify actionable interventions and associated metrics across the ME continuum seeking to use existing data for longitudinal tracking when possible. The document was shared and discussed with key stakeholders with iterative revisions to develop and assure plan engagement and support.

Results Four actionable EDI education-related categories were agreed upon: (1) purpose \& culture; (2) recruitment and retention; (3) curriculum and program structure; (4) evaluation and assessment (figure 1). Within each category 2-3 specific PDSA interventions were identified for action within 